\title{
Cryogenic UHV Specimen Preparation for APT: A Transfer Solution
}

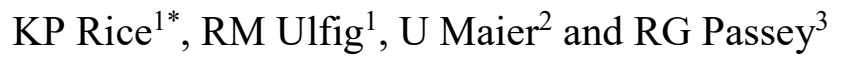 \\ 1. CAMECA Instruments Inc., Madison, WI, USA. \\ 2. Ferrovac GmbH, Zurich, Switzerland. \\ 3. Thermo Fisher Scientific, Hillsboro, OR, USA. \\ * Corresponding author: katherine.rice@ametek.com
}

As the application space for atom probe tomography (APT) expands, new hardware innovations are required to prepare and transfer specimens under cryogenic and/or ultra-high vacuum (UHV) conditions. For example, the analysis of biological materials, hydrogen containing materials [1], or surfaces prone to rapid oxidation, require that the specimen be maintained in a controlled environment from focused-ionbeam (FIB) manufacture through APT analysis. Designing hardware to successfully meet these application demands typically requires extensive experience in multiple areas of expertise, which may be beyond the typical laboratory $[2,3]$. Here we present a commercial cryo-UHV solution for FIB-APT that can be implemented to meet the transfer process requirements between FIB and APT for nonexperts.

The CAMECA ${ }^{\circledR}$ vacuum cryo-transfer-module (VCTM) is a portable, cryogenically cooled, UHV module that enables transfer and short-term storage of prepared specimens from DualBeam ${ }^{\mathrm{TM}}$ FIB-SEM systems to the atom probe [4]. Ferrovac, working in collaboration with Thermo Fisher Scientific and CAMECA, has engineered a Quick-Loader ${ }^{\mathrm{TM}}$-based system that attaches to a DualBeam chamber and enables transfer into the VCTM for transport to a LEAP ${ }^{\circledR} 5000$. The module or "suitcase" features a portable, battery-operated ion pump and a non-evaporable getter (NEG) to maintain UHV conditions, and a cold stage to maintain low-temperature conditions. The temperature and vacuum control prevent the formation of crystalline ice on the prepared samples, which would inhibit the subsequent APT analysis.

A primary consideration for transfer between FIB and APT is attachment of the VCTM to the FIB without compromising the FIB imaging resolution, due to vibrations from the attached suitcase while maintaining UHV conditions within the VCTM. Ferrovac's novel docking station, shown in Fig. 1a, was engineered meet all requirements for the transfer process. The docking system is installed using the standard mechanical and vacuum interface provided on Thermo Fisher Scientific instruments. The small loading-docking chamber is equipped with an O-ring sealed quick-connector that allows for a fast and simple attachment of the VCTM while maintaining the functionality of the standard sample loader on the DualBeam instrument. This is achieved by providing the ability to switch back and forth, between VCTM and a standard sample-loader transfer arm as illustrated in Fig. 1c.

The loading-docking chamber is pumped either via the existing roughing pump of the FIB or a separate scroll pump. Control of transfer gate-valve and pump-down-valve is established by a logic circuit interfacing with the FIB's standard DB15 port for fast loaders. Thus, the loading sequence of the FIB's software control can be applied without any software modification. Experiments performed with a VCTM have shown that UHV conditions in the VCTM are maintained after exposure to a vacuum level present in a standard FIB. Respective data is shown in Fig. 1d. 
References:

[1] Y-S Chen et al., Science 355 (2017), p. 1196.

[2] SSA Gerstl et al., Microscopy and Microanalysis 23 (2017), p. 612.

[3] DK Schreiber et al., Ultramicroscopy 194 (2018), p. 89.

[4] LT Stephenson et al., PLoS ONE 13 (2018), p. 1.
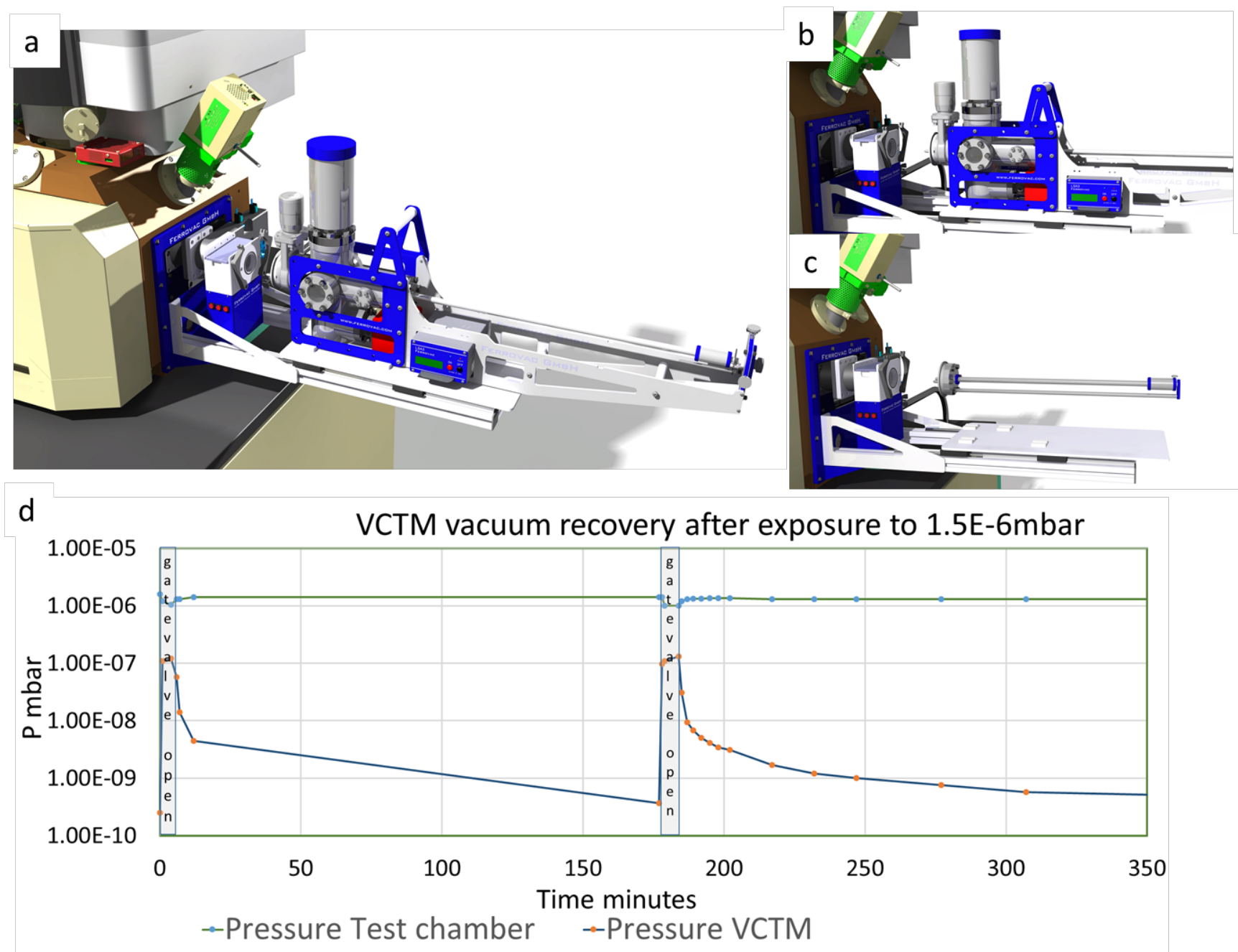

Figure 1. (a1) VCTM "UHV-Suitcase", (a2) loading chamber with quick connector, (a3) transfer arm, (a4) battery driven ion pump controller, (a5) LN2 Dewar, (a6) linear sledge.(b) Detached VCTM (c) VCTM replaced by standard transfer arm. (d) Exposure to simulated DualBeam vacuum level and recovery of VCTM base pressure. 